\title{
Validation of metabotypes identified in an Irish population in the German KORA FF4 study
}

\author{
Anna Riedl ${ }^{1,2}$, Nina Wawro ${ }^{1,2}$, Christa Meisinger ${ }^{1,2}$, Annette Peters ${ }^{3,4}$, Wolfgang Rathmann ${ }^{4,5}$, \\ Wolfgang Koenig ${ }^{6,7}$, Hannelore Daniel ${ }^{8}$, Hans Hauner ${ }^{9,10}$, Lorraine Brennan ${ }^{11}$ and \\ Jakob Linseisen ${ }^{1,2}$ \\ ${ }_{1}^{1}$ Independent Research Group Clinical Epidemiology, Helmholtz Zentrum München, German Research Center for \\ Environmental Health (GmbH), Neuherberg, Germany, \\ ${ }^{2}$ Chair of Epidemiology, Ludwig-Maximilians-Universität München, at UNIKA-T (Universitäres Zentrum für \\ Gesundheitswissenschaften am Klinikum Augsburg), Augsburg, Germany, \\ ${ }^{3}$ Institute of Epidemiology, Helmholtz Zentrum München, German Research Center for Environmental Health \\ ( GmbH), Neuherberg, Germany, \\ ${ }^{4}$ German Center for Diabetes Research (DZD e.V.), Neuherberg, Germany, \\ ${ }^{5}$ Institute for Biometrics and Epidemiology, German Diabetes Center, Leibniz Center for Diabetes Research at Heinrich \\ Heine University Düsseldorf, Düsseldorf, Germany, \\ ${ }^{6}$ Deutsches Herzzentrum München, Technical University of Munich, Munich, Germany, \\ ${ }^{7}$ Institute of Epidemiology and Medical Biometry, University of Ulm, Ulm, Germany, \\ ${ }^{8}$ Chair of Nutritional Physiology, Technical University of Munich, Freising-Weihenstephan, Germany, \\ ${ }^{9}$ Else Kröner-Fresenius Centre for Nutritional Medicine, Technical University of Munich, Freising-Weihenstephan, \\ Germany, \\ ${ }^{10}$ Institute of Nutritional Medicine, Klinikum rechts der Isar, Technical University of Munich, Munich, Germany and \\ ${ }^{11}$ Institute of Food and Health, UCD School of Agriculture and Food Science, UCD, Dublin, Ireland
}

\begin{abstract}
Previous work in an Irish cross-sectional study in adults identified three metabolic subgroups (metabotypes) of individuals using k-means cluster analysis based on four fasting clinical standard parameters (triacylglycerols, total cholesterol, HDL cholesterol and glucose). We aimed to validate these metabotypes in another large population-based study. We assigned 2221 participants aged 38-88 years from the German Cooperative Health Research in the Region of Augsburg (KORA) FF4 study (2013/2014) to the three metabotype clusters identified previously by minimizing the Euclidean distances. These clusters were characterized and compared with one another by metabolic characteristics as well as by cardiometabolic disease prevalence. Further, usual dietary intake of various foods/nutrients was estimated based on a food frequency questionnaire and multiple 24-hour food lists and was investigated across clusters. We identified three metabolically distinct clusters in the KORA FF4 study. Cluster 3 represented the group of participants with the most unfavorable metabolic characteristics (e.g. parameters of glucose and lipid metabolism, inflammatory markers), followed by clusters 2 and 1. Individuals in cluster 3 had the highest prevalence of metabolic diseases. Furthermore, they were characterized by the most unfavorable diet with significantly lowest intakes of vegetables, dairy products and fibers as well as significantly highest intakes of total, red and processed meat. Our finding of distinct metabolic subgroups in the KORA FF4 study suggest a successful validation of the metabotypes originally identified based on four commonly measured clinical parameters. Based on these metabotypes, targeted dietary recommendations may be developed for metabolic disease prevention.
\end{abstract}

\section{Conflict of Interest}

There is no conflict of interest 